\title{
Ultrafast adaptive optical near-field control
}

\author{
T. Brixner, ${ }^{1}$ F. J. García de Abajo, ${ }^{2}$ J. Schneider ${ }^{1}$ C. Spindler, ${ }^{1}$ and W. Pfeiffer ${ }^{1}$ \\ ${ }^{1}$ Physikalisches Institut, Universität Würzburg, Am Hubland, 97074 Würzburg, Germany \\ ${ }^{2}$ Centro Mixto CSIC-UPV/EHU, Apartado 1072, 20080 San Sebastián, Spain
}

(Received 21 October 2005; published 28 March 2006)

\begin{abstract}
Simultaneous control of the spatial and temporal properties of the optical near field in the vicinity of a nanostructure is achieved by illumination with broadband optimally polarization-shaped femtosecond light pulses. Here we demonstrate the spatial control of the local linear and nonlinear fluence, the local spectral distribution, and the local temporal intensity profile on a subdiffraction length scale. The boundary-element method is used for a self-consistent solution of Maxwell's equations in the frequency domain. Particular control objectives for spatial field distribution and temporal evolution are expressed as fitness functions in an evolutionary algorithm that optimizes adaptively the polarization-shaped input light pulses. Substantial control according to different goals is demonstrated and the limits of controllability are investigated. The dominating control mechanism is local interference of near-field modes that are excited with the two independent polarization components of the incident light pulses and hence polarization pulse shaping is essential to achieve substantial control in the optical near field. The influence of other control mechanisms is discussed and a number of applications are presented.
\end{abstract}

DOI: 10.1103/PhysRevB.73.125437

\section{INTRODUCTION}

The optical response of complex nanostructures exhibits fascinating properties, such as subwavelength variation of the field strength, local field enhancement with respect to the incident wave, and local fields with vector components perpendicular to those of the incident field. The subwavelength field strength variation is responsible for the spatial resolution obtained in scanning near-field optical microscopes (SNOM). ${ }^{1}$ Surface-enhanced Raman scattering from single molecules is at least partially attributed to the local field enhancement increasing the Raman scattering efficiency. ${ }^{2}$ The rapidly evolving capabilities of controlled nanostructure fabrication allows extensive tailoring of the optical response, thus leading to the emergence and rapid growth of nanoplasmonics. ${ }^{3}$ Phenomena such as enhanced transmission through subwavelength holes, ${ }^{4}$ or subwavelength-sized wave guides consisting of chains of spherical metal nanoparticles $^{5,6}$ all rely on the complex optical response of well-defined metal nanostructures.

The majority of optical near-field experiments is performed using narrow-bandwidth laser illumination or broadbandwidth incoherent radiation sources. However, it was shown recently that the combination of ultrafast laser spectroscopy, i.e., illumination with broadband coherent light sources, and near-field optics opens a new realm for nonlinear optics on the nanoscale. ${ }^{7-12}$ For example, local field enhancement and the detection of nonlinear optical signals improves the lateral resolution in SNOM, ${ }^{7}$ and secondharmonic microscopy from nanostructured metal films illustrates the intricate problems of local field enhancement and local nonlinear response. ${ }^{8}$ In these experiments the ultrashort laser pulses primarily serve to obtain a strong nonlinear response, whereas the exact spatial and temporal field distribution is of minor importance. This is no longer the case in time-resolved two-photon photoemission spectroscopy ${ }^{10-12}$ and SNOM imaging of plasmonic nanostructures. ${ }^{9}$ The study of time-resolved two-photon pho- toemission from supported silver nanoparticles shows that the optical near-field response and the corresponding temporal evolution of the local field determines the photoemission process. ${ }^{10}$ In addition, the experiments by Kim et al. demonstrate that the spatial field distribution contains valuable information, since it reflects details about the optical response of the nanostructure, such as the homogeneous linewidth of spectral resonances. ${ }^{9}$ More recently two-photon photoelectron emission microscopy (2P-PEEM) was applied to investigate the local field enhancement with spatial ${ }^{11}$ and temporal resolution. ${ }^{12}$

Ultrashort laser pulses excite the optical near field of the nanostructure over a range of frequencies and the superposition of these field distributions with different frequencies determines the actual local field evolution. Hence, the spectral phase of the illuminating laser pulses influences the resulting momentary field distribution in the vicinity of the nanostructure. This opens fascinating possibilities for the control of the optical near field as demonstrated in a theoretical study by Stockman et al. ${ }^{13}$ They showed that snapshots of the field distribution of a $V$ shaped and an arbitrarily shaped nanostructure exhibit distinct field patterns that vary as the linear chirp, i.e., the quadratic spectral phase, of the illuminating laser pulse is modified.

In general, interference phenomena provide means to achieve control over physical systems. First suggestions of coherent control schemes were made 20 years ago. ${ }^{14-19}$ In the meantime many experimental realizations have been demonstrated, for example in coherent quantum control of chemical reactions using adaptive femtosecond pulseshaping techniques (Ref. 20 and references therein). Coherent control requires at least two different excitation pathways, such that constructive versus destructive interference can be utilized. In experiments using ultrashort laser pulses usually many interfering pathways are excited simultaneously, thus leading to a controlled propagation of quantum-mechanical wave packets in space and time. The shape of the laser pulse can then be used to control the final- 


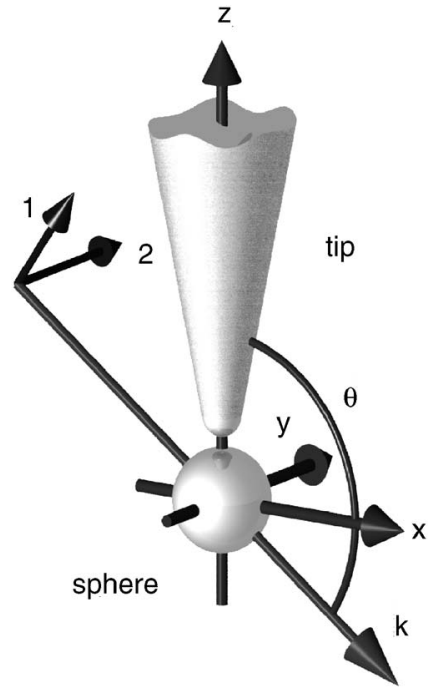

FIG. 1. Representation of the model nanostructure, Cartesian coordinate system, wave vector $\boldsymbol{k}$ of the incident light field, and the two orthogonal polarization components 1 and 2 . The truncated conical tip is not shown to its full length.

state amplitude. In many cases, the knowledge about the quantum-mechanical system is not sufficient to predict the optimal pulse shape, but it can be determined automatically using adaptive schemes and a learning algorithm. ${ }^{21}$ Recently the possibilities for pulse shaping were extended from phase and amplitude shaping to polarization pulse shaping. ${ }^{22-25}$ This was shown to extend the possibilities in time-resolved spectroscopies such as coherent anti-Stokes Raman spectrometry (CARS), ${ }^{26}$ and to allow new quantum control schemes in atoms ${ }^{27}$ and molecules. ${ }^{28-30}$

The application of polarization pulse shaping also allows significant control of optical near fields. We showed that polarization-shaped pulses can be used to control the evolution of the local electric field vector orientation and magnitude in three dimensions, i.e., field control of transverse and longitudinal components. ${ }^{31}$ More recently, we have proposed a scheme to utilize ultrafast optical near-field control for a new spatially and temporally resolved spectroscopy on the nanoscale in which ultrashort pump and probe excitations occur not only at different times but also at different positions with subdiffraction resolution. ${ }^{32}$ In the present paper we discuss the background of those simulations in detail (Sec. II), and we realize a number of (additional) control goals: local linear flux, local nonlinear flux, local spectrum, and space-time intensity evolution (Sec. III). In addition, we discuss the dominating control mechanisms (Sec. IV) and describe potential extensions and applications of polarization pulse shaping in near-field optics and ultrafast spectroscopy in the conclusion and outlook (Sec. V).

\section{METHODS AND SYSTEM CHARACTERISTICS}

\section{A. Boundary-element method and nanostructure}

The used model nanostructure is depicted in Fig. 1. It consists of a truncated conical metal tip with a tip radius of
$10 \mathrm{~nm}$, an opening angle of $5^{\circ}$ and a length of $1500 \mathrm{~nm}$. The tip apex is located $5 \mathrm{~nm}$ above a metal sphere of $25 \mathrm{~nm}$ radius that resides in the origin of the coordinate system. A plane wave illuminates the nanostructure. The illumination direction is indicated by the $k$ vector that lies in the $x-z$ plane and has the angle $\theta$ with the $z$ axis. Polarization pulse shaping requires two independent transverse polarization components in the incident wave. Here the components 1 and 2 are chosen to lie in the $x-z$ plane and parallel to the $y$ axis, respectively.

We solve the frequency-dependent Maxwell equations for a given nanostructure and incidence angle by means of the boundary-element method. ${ }^{33,34}$ In this Green's function approach, equivalent surface charges $\sigma_{j}$ and currents $\mathbf{h}_{j}$ are introduced on the boundary of each homogeneous region $S_{j}$ to account both for external sources and for induced sources beyond boundary surfaces. The electric field scattered by the boundaries inside each region $j$ is expressed in terms of scalar and vector potentials $\phi$ and $\mathbf{A}$ as $\mathbf{E}_{\text {scat }}(\mathbf{r}, \omega)=i k \mathbf{A}(\mathbf{r}, \omega)$ $-\nabla \phi(\mathbf{r}, \omega)$, where

$$
\begin{aligned}
& \phi(\mathbf{r}, \omega)=\int_{S_{j}} \frac{e^{i k \sqrt{\varepsilon_{j}(\omega)}|\mathbf{r}-\mathbf{s}|}}{|\mathbf{r}-\mathbf{s}|} \sigma_{j}(\mathbf{s}, \omega) d \mathbf{s}, \\
& \mathbf{A}(\mathbf{r}, \omega)=\frac{1}{c} \int_{S_{j}} \frac{e^{i k \sqrt{\varepsilon_{j}(\omega)}|\mathbf{r}-\mathbf{s}|}}{|\mathbf{r}-\mathbf{s}|} \mathbf{h}_{j}(\mathbf{s}, \omega) d \mathbf{s},
\end{aligned}
$$

and the integrals are extended over the closed surface limiting the noted region $j$. A self-consistent solution is obtained under the constraint that the usual electromagnetic boundary conditions are satisfied, using the boundary sources $\sigma_{j}$ and $\mathbf{h}_{j}$ as unknowns. The material properties are represented by a local, frequency-dependent dielectric function $\varepsilon_{j}(\omega)$ for each homogeneous region $j$. Here we use the bulk $\varepsilon(\omega)$ for gold and silver given from optical data. ${ }^{35}$ For the size of the nanostructures considered here, i.e., for particle sizes larger than $10 \mathrm{~nm}$, the use of the bulk $\varepsilon(\omega)$ is valid, since quantum size and surface effects can be neglected. ${ }^{36}$ The boundaryelement method reduces the electromagnetic problem to a set of surface-integral equations that are solved by discretization. The axial symmetry of the present model nanostructure allows to reduce the number of discretization steps by using a truncated basis of Bessel functions for Fourier decomposition. The number of discretization steps is set sufficiently high to guarantee convergence of the calculated fields.

As a result, we obtain complex-valued matrices $A_{\alpha}^{i}(\mathbf{r}, \omega)$, where the subscripts $\alpha=x, y, z$ indicate the component of the electric field at point $\mathbf{r}$ and frequency $\omega$ induced by a far field of superscripted linear polarization $i=1$ or $i=2$. The coefficients of this matrix are the local enhancement factors $A_{\alpha}^{i}(\mathbf{r}, \omega)$ for the electric field components, since their amplitude $\left|A_{\alpha}^{i}(\mathbf{r}, \omega)\right|$ describes the extent to which the two far-field components 1 and 2 couple to the local field component $E_{\alpha}(\mathbf{r}, \omega)$. Results for $\left|A_{\alpha}^{i}\left(\mathbf{r}, \omega_{0}\right)\right|$, with $\omega_{0}=2.46 \mathrm{rad} \mathrm{fs}^{-1}$, are shown for the $y-z$ plane in Fig. 2 . The density plots directly reflect the local enhancement of the field component with respect to the amplitude of the incident wave. Note that in general the orientation of the local field vector differs from 


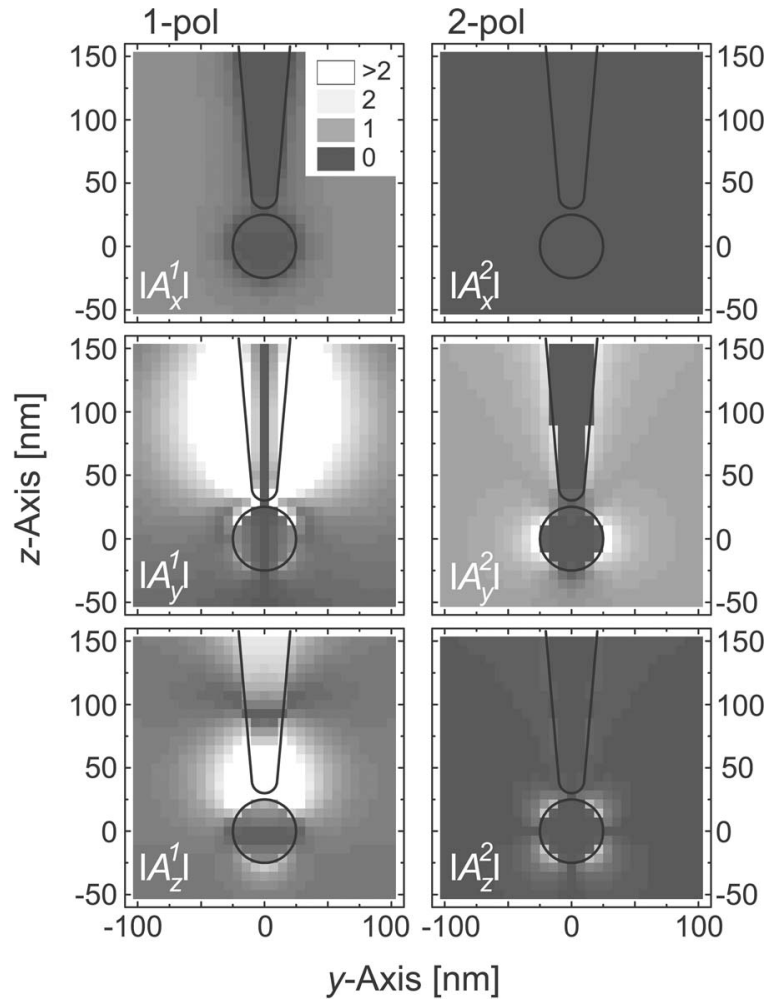

FIG. 2. Spatial field distribution. Density plots of the amplitude of the local response components $A_{\alpha}^{i}$ for illumination of a Ag nanostructure (see Fig. 1) at $767 \mathrm{~nm}$ for the $y-z$ plane. The wave vector orientation of the illuminating planar wave is given by $\theta=135^{\circ}$, i.e., the incident wave comes from the $-x$ direction and has an incidence angle of $45^{\circ}$ with respect to the $z$ axis. All plots are displayed on the same intensity gray scale, to allow a direct comparison of the enhancement factors. The black solid lines represent the cross sections of the tip and sphere.

the orientation of the incident field. For illumination with polarization 1 the field distribution is dominated by the $z$ component $E_{z}$, because of the antenna effect of the elongated tip. The maximum local field enhancement for $E_{z}$ of about 35 (truncated in Fig. 2) occurs between tip and nanoparticle. The region of this high enhancement is highly localized. It drops to 2 already $30 \mathrm{~nm}$ from the location of maximum $\left|A_{z}^{1}\right|$. Interestingly, a rather large enhancement of about 12 is seen for $\left|A_{y}^{1}\right|$ in the $y-z$ plane along the tip although the incident wave has no field component in this direction. We attribute this to the excitation of higher multipole plasmonic modes in the tip that exhibit multiple field nodes along the tip, similar to those reported for nanorods. ${ }^{37}$ For illumination with polarization 2 the local field enhancement is much smaller. The polarization 2 is parallel to the $y$ direction and hence an almost dipolar mode is excited in the metal sphere. The dipolar character is seen in the enhancement of $\left|A_{y}^{2}\right|$ at the sphere surface and the clover leaf shape of $\left|A_{z}^{2}\right|$ in the $y-z$ plane surrounding the sphere. For a $\mathrm{Au}$ nanostructure the field distribution (not shown here) looks very similar, i.e., the distributions have the same shape but slightly different enhancement factors. Accordingly, the near-field distribution induced by illumination with polarization 1 is dominated by the antenna effect of the tip, whereas it is dominated by the

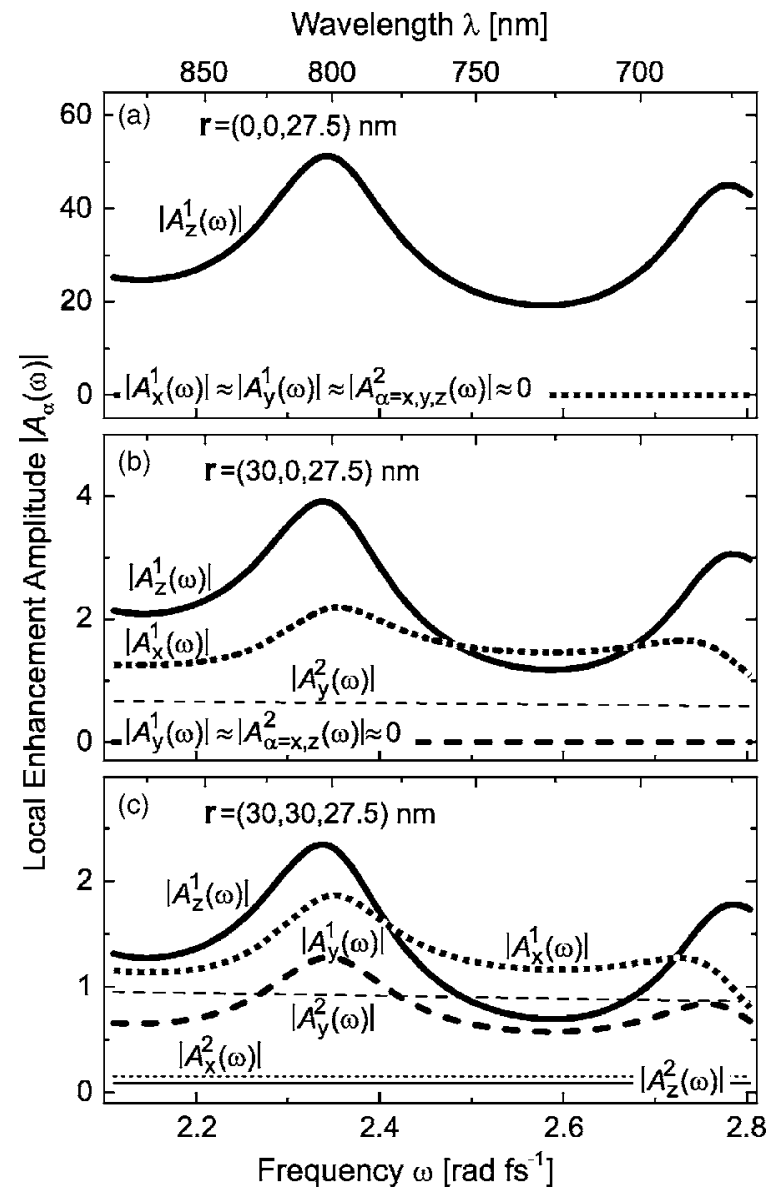

FIG. 3. Spectral distribution of the local enhancement amplitude $\left|A_{\alpha}^{i}\left(\mathbf{r}_{j}, \omega\right)\right|$ for different locations in the $z=27.5 \mathrm{~nm}$ plane for excitation with polarization 1 (thick lines) and polarization 2 (thin lines). The $x, y$, and $z$ components of the enhancement amplitude are represented by dotted, dashed, and solid lines, respectively. The calculation is performed for a $\mathrm{Ag}$ nanostructure with $\theta=135^{\circ}$.

response of the sphere for illumination with polarization 2 .

Since we illuminate the nanostructure with broadband polarization-shaped laser pulses for the purpose of near-field control, the spectral variation of the local response $A_{\alpha}^{i}(\mathbf{r}, \omega)$ must be considered. Figure 3 shows the spectral distribution of the local enhancement amplitude $\left|A_{\alpha}^{i}(\mathbf{r}, \omega)\right|$ for several locations $\mathbf{r}_{j}$ in the vicinity of the nanostructure. Directly under the tip apex the local field is dominated by the $z$ component, and local field enhancement factors between 20 and 50 are obtained [Fig. 3(a)] for 1-polarization excitation. The field components in $x$ and $y$ direction are negligible for this location. The spectral variation of the response is attributed to the excitation of plasmon polaritons in the tip, since the corresponding peaks are present also in the response of the tip alone. Multipole plasmon polaritons of different order explain the occurrence of two maxima in the response function. The multipole plasmon polariton is a standing wave and thus the spectral position of higher order modes is influenced by the chosen tip length. Here, the length is chosen large enough to shift the second-order mode beyond the longwavelength limit of the excitation spectrum. Accordingly, the truncation of the tip and the related spectral structure in the 
response function only marginally influence the results presented below. The spectral response of the Ag nanosphere alone is dominated by the plasmon polariton located at a frequency of $5.22 \mathrm{rad} \mathrm{fs}^{-1}(360 \mathrm{~nm})$. For a small nanoparticle multipole plasmons are negligible and thus the nanoparticle response does not lead to spectral features in the frequency range considered here. For excitation with polarization 2 the local field strength is negligible, since the tip cannot act as an antenna. At the location $(30,0,27.5) \mathrm{nm}$ the maximum field enhancement is strongly reduced [Fig. $3(b)]$. Still the $y$ component is zero because of symmetry reasons. In contrast, for 2-polarization excitation only the $y$ component is nonvanishing. Note that the nonvanishing field components exhibit similar strength for both polarization directions. This gives a handle for control by interference of the near-field modes (see Sec. IV A). At the off-axis position $(30,30,27.5) \mathrm{nm}$ all field components are symmetry allowed [Fig. 3(c)].

For a given incident field, which is characterized by the intensity distributions $I_{i}(\omega)$ and the corresponding phases $\varphi_{i}(\omega)$ for the two polarizations $i=1,2$, the total local field at location $\mathbf{r}$ is then obtained as

$$
\mathbf{E}_{\mathrm{local}}(\mathbf{r}, \omega)=\sum_{i=1}^{2}\left(\begin{array}{l}
A_{x}^{i}(\mathbf{r}, \omega) \\
A_{y}^{i}(\mathbf{r}, \omega) \\
A_{z}^{i}(\mathbf{r}, \omega)
\end{array}\right) \sqrt{I_{i}(\omega)} e^{i \varphi_{i}(\omega)} .
$$

Since this procedure delivers amplitudes as well as phases, the local field in the time domain $\mathbf{E}(\mathbf{r}, t)$ is easily calculated by separately Fourier transforming each vector component of $\mathbf{E}(\mathbf{r}, \omega)$.

\section{B. Polarization pulse shaping}

Femtosecond laser pulse shaping has become a wellestablished technology in recent years. ${ }^{38}$ Pulse shapers often involve a zero-dispersion compressor which spatially separates and recollimates the broadband laser spectrum. In the Fourier plane of highest optical resolution, a spatial light modulator (SLM) is placed as an active element that modulates the phase and/or amplitude of individual frequency components. Common SLM types are liquid-crystal displays (LCDs), acousto-optic modulators (AOMs), or deflectable mirrors (DMs). In the case of LCDs they have typical frequency resolutions of 128 or 640 independent pixels. This leads to a vast number of different possible temporal intensity and phase profiles of the electric field.

We have recently developed the technique of femtosecond phase and polarization shaping, ${ }^{22-25}$ based on a two-layer LCD in which each layer manipulates the spectral phase of one of the two orthogonal transverse polarization components. As a result, not only the intensity and phase but also the polarization state of light, i.e., its degree of ellipticity as well as orientation with respect to the laboratory reference frame, can be changed as functions of time within a single femtosecond laser pulse. This opens new prospects in the control of light-matter interaction since vector properties can be exploited. ${ }^{26-30}$ As will be shown below, this is also true in the present scheme of optical near-field control where polar- ization shaping is an essential ingredient. The maximum rates at which the polarization-state angles of ellipticity and orientation can be changed are equal to the laser frequency bandwidth. ${ }^{24}$ For the simulations here we assume Gaussian spectra (central frequency $\omega_{0}=2.46 \mathrm{rad} \mathrm{fs}^{-1}$, FWHM $=0.23 \mathrm{rad} \mathrm{fs}^{-1}$ ) and 128 frequency sampling points for both components. Hence, polarization properties can be changed on a time scale of the bandwidth-limited pulse duration, i.e., with approximately $15 \mathrm{fs}$ resolution. We consider an ideal situation with phase-only modulations of the two polarization components that leave the spectral shapes invariant. Additional polarization-state modifications by optical elements after passage through the pulse shaper are not considered here but have to be taken into account in a real experiment. ${ }^{23}$ The incidence angle of these (plane wave) modulated pulses on the nanostructure is as defined in Fig. 1.

\section{Closed-loop optimization}

The combination of far-field polarization shaping and near-field response as described in the previous two sections yields the local electric field at any position $\mathbf{r}$ via Eq. (3). We then use this quantity to define a number of different observables in analogy with far-field optics: local linear flux

$$
F_{l i n}(\mathbf{r})=\int_{-\infty}^{\infty} \sum_{\alpha=x, y, z} b_{\alpha}^{2} E_{\alpha}^{2}(\mathbf{r}, t) d t,
$$

local nonlinear flux

$$
F_{n o n l}=\int_{-\infty}^{\infty}\left(\sum_{\alpha=x, y, z} b_{\alpha}^{2} E_{\alpha}^{2}(\mathbf{r}, t)\right)^{2} d t,
$$

local spectrum

$$
S(\mathbf{r}, \omega)=\sum_{\alpha=x, y, z} b_{\alpha}^{2} E_{\alpha}^{2}(\mathbf{r}, \omega)=\sum_{\alpha=x, y, z} b_{\alpha}^{2}\left|\mathrm{FT}\left[E_{\alpha}(\mathbf{r}, t)\right]\right|^{2},
$$

and local time-dependent intensity

$$
I(\mathbf{r}, t)=\sum_{\alpha=x, y, z} b_{\alpha}^{2} E_{\alpha}^{2}(\mathbf{r}, t) .
$$

The parameters $b_{\alpha}$ describe which polarization components are included in the observable. Setting $b_{x}=1$ and $b_{y}=b_{z}=0$, for example, describes field-matter interaction with transition dipoles oriented along the $x$ axis.

The optimization procedure is illustrated in Fig. 4. Specific optimization goals of field distributions are expressed in terms of the observables from Eqs. (4)-(7) within a cost function (fitness function) $f$. An evolutionary algorithm ${ }^{39}$ adjusts the spectral phases $\varphi_{i}(\omega)$ of the two input polarization components in order to minimize (maximize) $f$. Using 128 LCD pixels and $1^{\circ}$ phase resolution, the search space contains $360^{128} \approx 10^{327}$ possible pulse-shaper configurations. In this context, the frequency-domain boundary-element method is an extremely powerful technique for obtaining local electric fields because the computationally expensive response calculation has to be performed only once for any given nanostructure; specifically shaped input fields can be considered by simple multiplication and one fast Fourier 


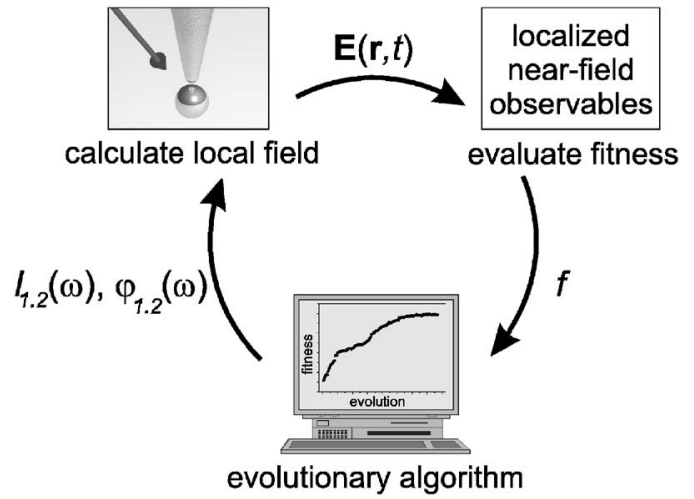

FIG. 4. Optimization loop. It contains the steps of calculating the near-field for a given geometry, evaluating a fitness function that describes how well a particular objective is reached, and iteratively improving the external laser-field parameters by an evolutionary learning algorithm.

transformation. This makes it possible to achieve convergence even in the large parameter space. Typically, we use 60 individuals per generation and a total of 300 generations for one optimization goal. Repetitive optimizations in general yield the same final fitness and almost identical optimal phase functions.

\section{ADAPTIVE NEAR-FIELD CONTROL}

\section{A. Control of local linear flux}

The first objective of near-field control considered here is the spatial localization of linear flux, i.e., time-integrated local intensity as defined in Eq. (4). The definition of a suitable fitness function is one of the key points in optimal control. We would like to achieve high flux at some target point $\mathbf{r}_{i}$ but low flux at "all" other points $\mathbf{r}_{n}$. Therefore, the straightforward approach would be to use the flux at the target position in the numerator, and the sum of fluxes at all other locations in the denominator, of the fitness function $f$ to be maximized. However, according to Fig. 2 there will be a spatially finite region within which the local field does not vary substantially (in the here considered nanostructure geometry and wavelength range this spatial spread is on the order of $20 \mathrm{~nm}$ ). It would hence not make sense to penalize too strongly any high flux at locations close to the target $\mathbf{r}_{i}$, as any high flux at the target will necessarily also increase the flux close by. Instead, we introduce a weighting factor 1 $-g\left(\mathbf{r}_{n}-\mathbf{r}_{i}\right)$ for all penalty terms, where

$$
g\left(\mathbf{r}_{n}-\mathbf{r}_{i}\right)=e^{-4 \ln 2\left(\left|\mathbf{r}_{n}-\mathbf{r}_{i}\right|^{2}\right) / w_{g}^{2}}
$$

such that the penalty weight increases with distance from the target point $\mathbf{r}_{i}$. Points further away from the target (i.e., those fields that according to the near-field properties may be

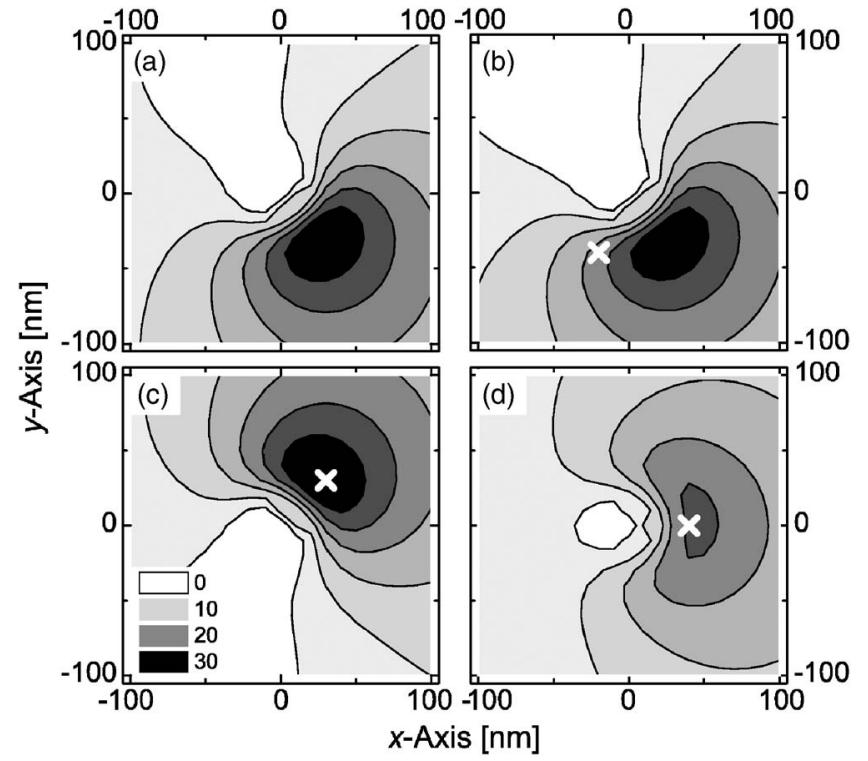

FIG. 5. Linear flux distributions $F_{\text {lin }}(\mathbf{r})$ for the $x$ and $y$ component of the field $\left(b_{x}=b_{y}=1\right.$ and $\left.b_{z}=0\right)$. A Ag nanostructure in the $z=27.5 \mathrm{~nm}$ plane is illuminated with (a) an unshaped laser pulse and (b)-(d) optimally shaped laser pulses using the fitness function given in Eq. (9) and the target locations indicated by the white crosses. The flux distribution is identically scaled in all parts to allow a direct comparison. The difference between neighboring contour lines is 5 arbitrary units of linear flux.

controlled independently) all receive the same penalty weight as the Gaussian decays to zero. The fitness is therefore evaluated as

$$
f\left[\varphi_{1}(\omega), \varphi_{2}(\omega)\right]=\frac{\int_{-\infty}^{\infty} \sum_{\alpha=x, y, z} b_{\alpha}^{2} E_{\alpha}^{2}\left(\mathbf{r}_{i}, t\right) d t}{\sum_{n \neq i}\left[1-g\left(\mathbf{r}_{n}-\mathbf{r}_{i}\right)\right] \int_{-\infty}^{\infty} \sum_{\alpha=x, y, z} b_{\alpha}^{2} E_{\alpha}^{2}\left(\mathbf{r}_{n}, t\right) d t} .
$$

In this example, we consider the $x$ and $y$ components of the electric field $\left(b_{x}=b_{y}=1\right.$ and $\left.b_{z}=0\right)$, and the sum over locations $\mathbf{r}_{n}$ is taken on a quadratic grid of 441 points in the plane defined by $z=27.5 \mathrm{~nm}$, with point separations of $10 \mathrm{~nm}$ in both directions. The Gaussian width $w_{g}$ of Eq. (8) is $20 \mathrm{~nm}$.

Results for the spatial $x-y$ distribution of linear flux in the target plane are shown in Fig. 5 for illumination with an unshaped laser pulse [Fig. 5(a)] and for optimal polarizationshaped laser pulses for three different target points [Figs. 5(b)-5(d)]. Illumination with an unshaped laser pulse, i.e., light that is polarized along the bisector of the angle between polarization 1 and 2, creates a flux distribution that is centered in the lower right corner of the $x-y$ plane and that is asymmetric with respect to the incidence plane ( $x-z$ plane). This asymmetry is attributed to the fact that the incident polarization along the diagonal between polarization 1 and 2 breaks the symmetry. By polarization shaping the flux maximum can now be shifted to the upper right corner of the $x-y$ plane $[$ Fig. $5(\mathrm{c})]$ and to the right of the origin of the plane 
[Fig. 5(d)]. The corresponding target point locations are indicated by crosses and it is obvious that the optimization goal is met. Hence, by using adaptive polarization shaping the position of the flux maximum can indeed be manipulated on the nanoscale. However, the controllability is limited, i.e., it is possible to optimize flux maximum only for positive $x$ values and target points that are located neither too close nor too far from the origin of the plane. The first limitation is demonstrated in Fig. 5(b). Here the target point is located in the left half of the $x-y$ plane whereas the corresponding optimized linear flux distribution has its maximum still at a positive value of $x$. Summarizing, best control can be achieved in a sickle-shaped area in the right half of the $x-y$ plane. We emphasize that this type of control does not result from specific spatial properties of the incoming laser beam, which was rather modeled as an (infinite) plane wave. The effects of additional focusing on near-field control will be presented elsewhere ${ }^{40}$ near-field control here is based entirely on the spectral-temporal properties of the polarizationshaped laser pulse, exploiting the near-field response of the nanostructure. Control mechanisms will be discussed in Sec. IV.

\section{B. Control of local nonlinear flux}

In this section, we discuss spatial control of second-order nonlinear signals, i.e., the characteristic field dependence in processes such as two-photon absorption (e.g., for subwavelength lithography), two-photon photoemission, or secondharmonic generation. Again a time-integrated quantity is considered [Eq. (5)]. This emphasizes that control is not just a transient phenomenon but that its effects can be detected even without time resolution. The fitness function is defined in analogy with the previous section, again using a distance weighting factor on the penalty terms for the nontargeted points, but employing the intensity squared as in Eq. (5) in the fitness function. Results for a number of target points are shown in Fig. 6.

Significant control over the position of nonlinear flux is achieved at high spatial contrast ratios. Lateral extensions of high-flux regions are on the order of $20 \mathrm{~nm}$ (FWHM), roughly determined by the size of the nanostructure and the length scale on which the near field changes its phase and amplitude. These results indicate that the location of nonlinear light-matter interaction can be positioned on a nanometer length scale without mechanical movement of the near-fieldgenerating nanostructure. Similar to the optimization of the local linear flux the controllability is limited to the right half of the $x-y$ plane.

The spatial range of achievable target positions is given by the maximum extension of the near-field modes, since they provide the mechanism for subdiffraction controllability. In the geometry investigated here, this accessible range has an approximate radius of $100 \mathrm{~nm}$ in the $x-y$ plane. Optimum controllability is achieved in those locations within this radius where the near-field modes excited by the two orthogonal input polarization components interfere maximally (see Sec. IV). Considering the near-field vector properties as shown in Fig. 2 and their relative phase (not shown), best

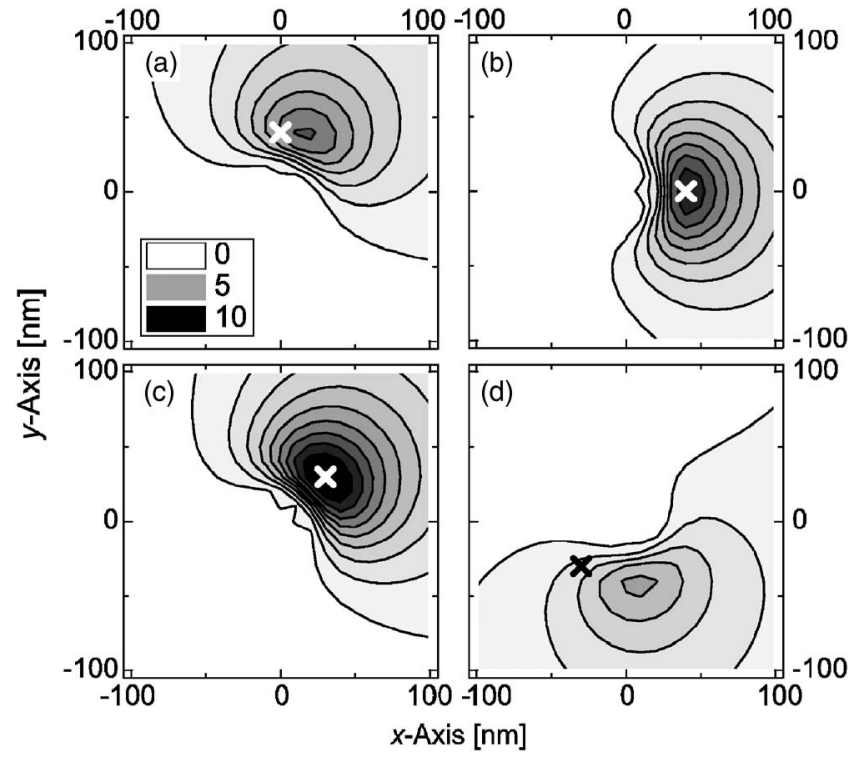

FIG. 6. Nonlinear flux distributions $F_{\text {nonl }}(\mathbf{r})$ for the $x$ and $y$ component of the field $\left(b_{x}=b_{y}=1\right.$ and $\left.b_{z}=0\right)$ in the $z=27.5 \mathrm{~nm}$ plane. A Ag nanostructure is illuminated with optimally shaped laser pulses and the target locations indicated by the crosses. The flux distribution is identically scaled in all parts to allow a direct comparison. The difference between neighboring contour lines is 1 arbitrary unit of nonlinear flux.

control over the $x-y$ field components is achieved in a sicklelike area in the right side of the $x-y$ plane,,$^{41}$ as it was also observed for the optimization of the linear flux. Directly in the center under the tip, the $z$ field component dominates the response and hence controllability vanishes. Thus, the optimization of the nonlinear flux exhibits similar limitations as for the linear flux.

The spatial steering demonstrated in Fig. 6 can be viewed as a nanoscale optical switching or multiplexing device. Tightly confined "light packets" can be delivered to different spatial "addresses" with a switching time that is limited only by the time scale on which the external pulse shapes can be changed. It is not necessary to modify the properties of the switching device (i.e., the nanostructure) in order to reach a different position; rather the desired "address" is directly encoded in the illuminating light itself. Hence the "processing time" of the multiplexer is limited only by the spectral bandwidth and the finite dephasing time of the given nanostructure and thus signals arrive at the desired targets immediately.

\section{Control of the local spectral distribution}

Apart from controlling integrated flux quantities as in the previous two sections, it is also possible to manipulate the spectral properties of the electromagnetic field on a nanometer length scale. As a demonstration, we chose to create locally "red-enhanced" and "blue-enhanced" spectra at two positions separated by $\Delta y=60 \mathrm{~nm}$. The fitness function employed here is 


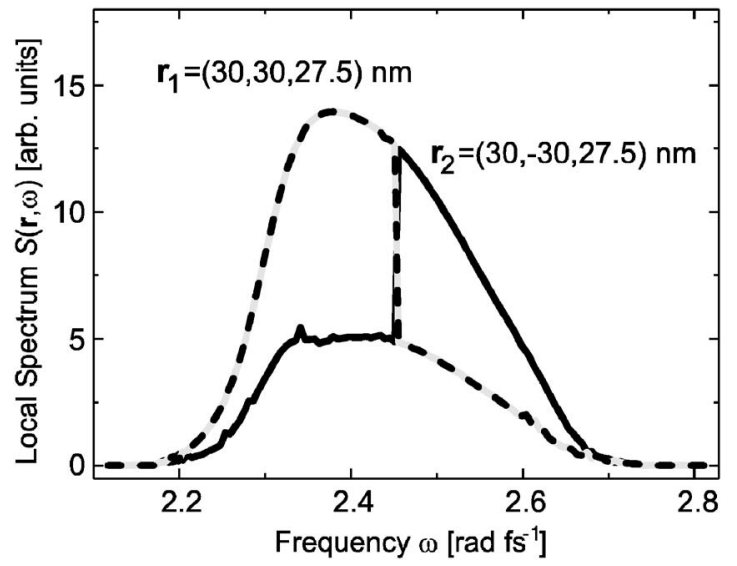

FIG. 7. Local spectra $S(\mathbf{r}, \omega)$ at the locations $\mathbf{r}_{1}$ $=(30,30,27.5) \mathrm{nm}$ (dashed line) and $\mathbf{r}_{2}=(30,30,27.5) \mathrm{nm}$ (solid line). A $\mathrm{Au}$ nanostructure is illuminated $\left(\theta=135^{\circ}\right)$ with a polarization-shaped laser pulse optimizing the fitness function defined in Eq. (10)

$f\left[\varphi_{1}(\omega), \varphi_{2}(\omega)\right]=\frac{\int_{0}^{\omega_{c}} S\left(\mathbf{r}_{1}, \omega\right) d \omega}{\int_{\omega_{c}}^{\infty} S\left(\mathbf{r}_{1}, \omega\right) d \omega} \cdot \frac{\int_{\omega_{c}}^{\infty} S\left(\mathbf{r}_{2}, \omega\right) d \omega}{\int_{0}^{\omega_{c}} S\left(\mathbf{r}_{2}, \omega\right) d \omega}$,

where $S(\mathbf{r}, \omega)$ are the local spectra as defined in Eq. (6) with $b_{x}=b_{y}=b_{z}=1$, and $\omega_{c}=2.46 \mathrm{rad} \mathrm{fs}^{-1}$ is a "cutoff" frequency, in this case the center frequency of the input spectrum. Best near fields are those which achieve high local spectral intensities for the "red" frequencies below $\omega_{c}$ at position $\mathbf{r}_{1}$ $=(30,30,27.5) \mathrm{nm}$, and simultaneously high local intensities for the "blue" frequencies above $\omega_{c}$ at position $\mathbf{r}_{2}=(30$, $-30,27.5) \mathrm{nm}$. Light intensity in the undesired spectral ranges above $\omega_{c}$ at $\mathbf{r}_{1}$ and below $\omega_{c}$ at $\mathbf{r}_{2}$ reduces the fitness value $f$.

The local spectra at the two target locations $\mathbf{r}_{1}$ and $\mathbf{r}_{2}$ after optimization are shown in Fig. 7. The spectral weight at $\mathbf{r}_{1}$ is shifted to the red whereas it is shifted to the blue at $\mathbf{r}_{2}$, thus the optimized near field shows indeed the desired characteristics. Figure 8 displays the variation of the local spectrum along the $y$ axis for $x=30 \mathrm{~nm}$. The low- and high-frequency regions at the two target locations $\mathbf{r}_{1}$ and $\mathbf{r}_{2}$ are indicated by rectangles. At $y=20 \mathrm{~nm}$, the low-frequency components dominate whereas the high-frequency components occur preferentially at $y=-20 \mathrm{~nm}$. It is possible to create nanolocalized "red" and "blue" light fields via polarization pulse shaping. In contrast, phase shaping of a linearly polarized laser pulse alone does not allow to optimize the local spectrum.

Note that control can be achieved independently for each frequency component since the optimized spectra exhibit a steplike behavior at $\omega_{c}$. This is attributed to the fact that control is possible via the interference of each frequency component and thus can be controlled independently (compare Sec. IV). Consequently, control of spectral properties is not limited to just one discriminating cutoff frequency. Complex local spectral shapes can be realized (results not shown)

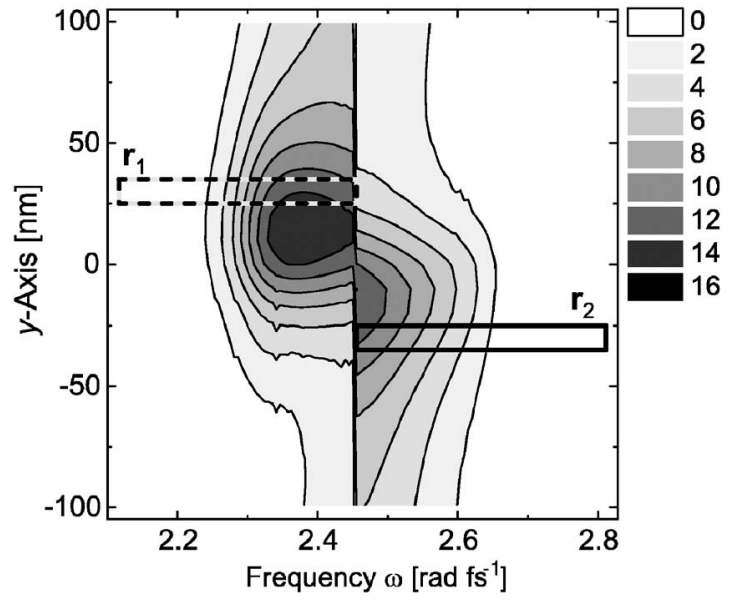

FIG. 8. Spatial variation of the spectral distribution $S(\mathbf{r}, \omega)$ along the $y$ axis for $x=30 \mathrm{~nm}$ in the $z=27.5 \mathrm{~nm}$ plane as contour plot for the optimization shown in Fig. 7. The low- and highfrequency regions at the two target locations $\mathbf{r}_{1}$ and $\mathbf{r}_{2}$ are indicated by rectangles.

by defining the appropriate target/fitness functionals with several spectral enhancing/suppressing regions. This technique transfers far-field pulse-shaping capabilities to the near-field region with nanoscale spatial resolution and variability.

\section{Spatio-temporal control}

One of the most general near-field control objectives is spatial-temporal shaping in which the target functional specifies the complete field evolution at different positions. We have discussed this type of near-field shaping previously, ${ }^{32}$ focusing on its application as a unique spectroscopic technique with simultaneous nanoscopic spatial and ultrafast temporal resolution. Here we will only show the main result of that study and additionally compare it with attempted control using linearly polarized light (without polarization shaping).

The desired spatial and temporal "focusing" is expressed in terms of the local time-dependent intensity [Eq. (7)] within the cost function

$$
\begin{aligned}
f\left[\varphi_{1}(\omega), \varphi_{2}(\omega)\right]= & \sum_{j} \int_{-\infty}^{\infty}\left[\left(\sum_{\alpha=x, y, z} b_{\alpha}^{2} E_{\alpha}^{2}\left(\mathbf{r}_{j}, t\right)\right)\right. \\
& \left.\times\left(\sum_{\alpha=x, y, z} b_{\alpha}^{2} E_{\alpha}^{2}\left(\mathbf{r}_{j}, t_{j}\right)\right)^{-1}-p_{j}(t)\right]^{2} d t, \\
& j=1,2,3, \ldots,
\end{aligned}
$$

where the $b_{\alpha}$ again determine which field components are optimized, and where $p_{j}(t)$ contains the temporal target functions at points $\mathbf{r}_{j}$. Investigating pulse-like targets at specific locations, we defined

$$
p_{j}(t)=e^{-4 \ln 2\left[\left(t-t_{j}\right)^{2} / \tau_{j}\right]}
$$

as a normalized Gaussian distribution centered at $t_{j}$ with an FWHM of $\tau_{j}$. We select $\tau_{j}$ equal to the bandwidth-limited 
pulse duration of the external laser such that we generate ultrashort pulses located at the specified time and place. For each position $\mathbf{r}_{j}$, a different target time $t_{j}$ can be chosen. The cost functional is then given by the squared deviation of the desired from the actual intensity profile, integrated over time and summed over all investigated points. The factor $\left[\Sigma_{\alpha} b_{\alpha}^{2} E_{\alpha}^{2}\left(\mathbf{r}_{j}, t_{j}\right)\right]^{-1}$ normalizes the actual local intensity to its value at the peak position $t_{j}$. Minimization of $f$ by the evolutionary algorithm then leads to the overall best possible realization of the desired field properties.

We have conducted two types of external field optimization: polarization and phase shaping (i.e., phase shaping for two orthogonal input polarization components as in all the previous sections) as well as phase-only shaping of one linear polarization component. The latter was achieved by restricting $\varphi_{1}(\omega)=\varphi_{2}(\omega)$ in the optimization algorithm. This coupling of both phase modulations leads to a purely linear polarization along the diagonal between the 1 and 2 coordinate axes, and therefore allows the scheme to excite both the 1-pol and 2-pol near-field components discussed in Fig. 2, although these two components may not be addressed independently. The target in both the polarization and phase as well as the phase-only optimization was to achieve high intensity (using $b_{x}=b_{y}=1$, and $\left.b_{z}=0\right)$ at point $\mathbf{r}_{1}=(20$, $-20,27.5) \mathrm{nm}$ and time $t_{1}=0$ fs as well as high intensity at point $\mathbf{r}_{2}=(20,20,27.5) \mathrm{nm}$ and time $t_{2}=40$ fs with pulse durations $\tau_{1}=\tau_{2}=15 \mathrm{fs}$, but low intensity at all other times. This would correspond to a spatial-temporal pump-probe scheme in which the pump pulse excitation is localized at a different time and at a different place than the probe excitation.

The results of these optimizations for a Au nanostructure are shown in Fig. 9. For polarization and phase modulation, the temporal intensity evolution indeed shows the desired behavior [Fig. 9(a)]. At position $\mathbf{r}_{1}$ (solid line) the main peak occurs at time $t=0 \mathrm{fs}$, and at position $\mathbf{r}_{2}$ (dashed line) the light is concentrated around $t=40 \mathrm{fs}$, with much reduced intensity at the undesired locations. The complete time-space evolution is shown as a function of the $y$ coordinate in Fig. 9(b). The maximum of the field distribution clearly switches from negative $y$ coordinates at $t=0$ fs to the positive side at the time of the second pulse. Similar to the previous sections, spatial resolution in both $x$ and $y$ directions is on the order of $30 \mathrm{~nm}$. The quality of spatio-temporal control depends on the exact location of the target points and on which field components are included ( $b_{\alpha}$ factors). This explains why Fig. 9(a) shows an even better contrast ratio between "pump" and "probe" excitation than the results shown in our previous work. ${ }^{32}$ In that work it was also shown that the time separation between the spatially localized pump and probe excitations can be varied at will within the time window of the pulse shaper. ${ }^{32}$ It is also possible to modify the pulse durations individually by changing the target widths $\tau_{j}{ }^{32}$ Overall, space-time-resolved creation of electromagnetic near fields is possible in a very general sense and opens the perspective for unique types of spectroscopy (Sec. V).

For comparison, the result of the phase-only optimization with the same spatial-temporal target is shown in Figs. 9(c) and $9(\mathrm{~d})$. Here the spatial symmetry cannot be broken (in
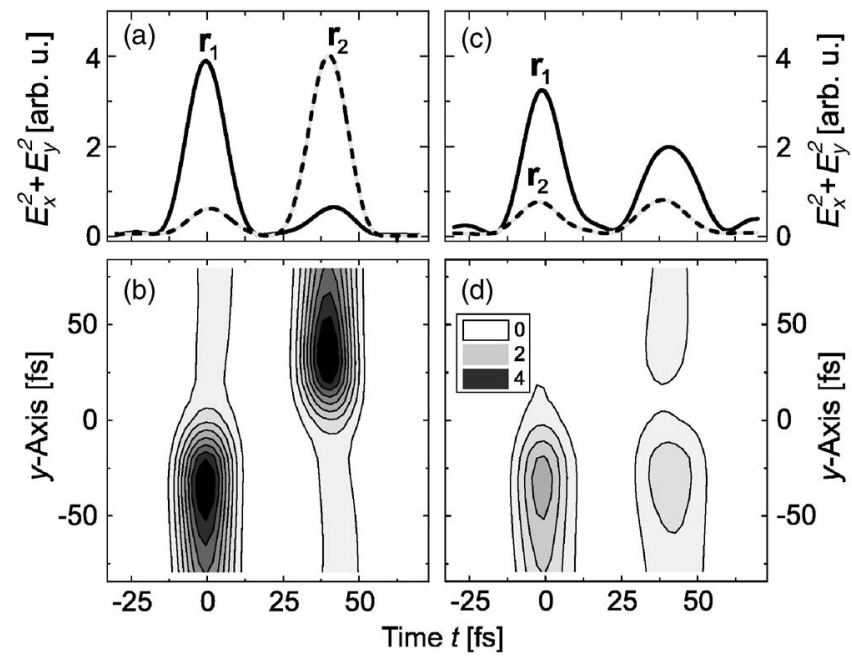

FIG. 9. Spatio-temporal localization of the optical near-field intensity $I(\mathbf{r}, t)$. The temporal evolution of the intensity of the $x$ and $y$ components of the local electric field is shown for an optimally polarization and phase shaped laser pulse (left) and linearly polarized phase-shaped laser pulse with the polarization vector oriented along the diagonal between polarization 1 and 2 (right). The graphs (a) and (c) show the intensity evolution at $\mathbf{r}_{1}=(20,-20,27.5) \mathrm{nm}$ and at $\mathbf{r}_{2}=(20,20,27.5) \mathrm{nm}$ as solid and dashed lines, respectively. The two lower graphs (b) and (d) represent the temporal evolution of the intensity along the straight line defined by $\mathbf{r}_{1}$ and $\mathbf{r}_{2}$ as contour plots.

contrast to the polarization-modulated example), and the peak intensities of the two pulses are nearly the same for any of the positions $\mathbf{r}_{1}$ or $\mathbf{r}_{2}$ [Fig. 9(c)]. This is also evident from the contour plot [Fig. 9(d)], showing a light localization for negative $y$ coordinates only, instead of the desired switching behavior. We conclude from this failure of the optimization that polarization shaping is an essential ingredient in spatialtemporal near-field control. Although with phase-only shaping and the correct polarization direction, it is also possible to excite the same near-field modes as with polarization shaping, they cannot be addressed independently. As will be seen in the next section, it is exactly the possibility for individually controlled mode superposition that makes near-field control possible. Although some degree of control is still obtainable with chirp effects, the main mechanism requires polarization modulation.

\section{CONTROL MECHANISMS}

\section{A. Interference of near-field modes}

The results from Sec. III demonstrate that a number of properties of optical near fields can be controlled in a very general sense. Specific manipulation of observables is possible on a sub-diffraction-limited length scale, even though the externally polarization-shaped laser field was modeled as a plane wave without any transverse spatial variation. One of the main control mechanisms is local interference of nearfield modes as will be explained now.

It was seen in Fig. 2 that in certain spatial regions the near-field mode components $(x, y$, and $z$ ) have nonvanishing 


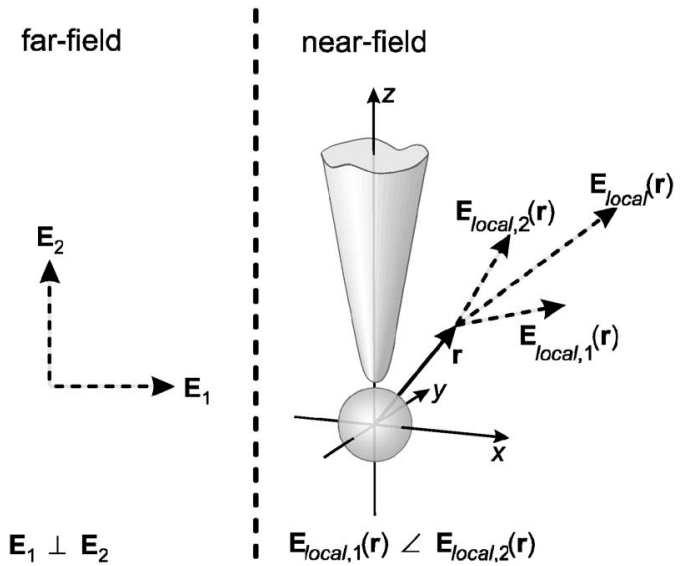

FIG. 10. Local interference scheme. The local fields $\mathbf{E}_{\text {local, } 1}$ and $\mathbf{E}_{\text {local }, 2}$ excited by the orthogonal external fields $\mathbf{E}_{1}$ and $\mathbf{E}_{2}$ are in general not orthogonal and interfere.

strength both for 1-polarized as well as 2-polarized excitation. This can lead to interference between the 1-excited and the 2-excited fields. An intuitive simplified illustration is presented in Fig. 10. Consider for now just one frequency component. In the far field, polarization directions 1 and 2 are perpendicular to each other as they represent the two independent transverse components of the incident laser field. Because of this orthogonality, there is no interference between them that could change the total intensity $\left|\mathbf{E}_{1}+\mathbf{E}_{2}\right|^{2}$ $=\left|\mathbf{E}_{1}\right|^{2}+\left|\mathbf{E}_{2}\right|^{2}$ upon variation of the phases $\varphi_{1}$ or $\varphi_{2}$.

However, the situation is different in the optical near field. At one particular location, the electric field vector excited by far-field component $\mathbf{E}_{1}$, labeled $\mathbf{E}_{\text {local }, 1}$, is in general not parallel to $\mathbf{E}_{1}$ because of the introduction of additional field components (both transverse and longitudinal), as seen in Eq. (3) and Fig. 2. Similarly, the local field $\mathbf{E}_{\text {local, }}$ generally points into a different direction than the external field $\mathbf{E}_{2}$ by which it is excited. As a consequence, the two local fields $\mathbf{E}_{\text {local }, 1}$ and $\mathbf{E}_{\text {local }, 2}$ are not orthogonal to each other and therefore can interfere. Now the total intensity $\mid \mathbf{E}_{\text {local }, 1}$ $+\left.\mathbf{E}_{\text {local, } 2}\right|^{2}$ and observable quantities such as those defined in Eqs. (4)-(7) depend both on the relative orientation of the two near-field vectors as well as on their mutual phase relation. Constructive interference versus destructive interference can be used for local intensity enhancement or suppression, respectively.

The phases of the local electric fields $\mathbf{E}_{\text {local }, 1}$ and $\mathbf{E}_{\text {local, } 2}$ are determined by two factors according to Eq. (3): the externally applied phases $\varphi_{1}(\omega)$ and $\varphi_{2}(\omega)$ as well as the phases of the near-field response $\arg \left\{A_{\alpha}^{i}(\mathbf{r}, \omega)\right\}$. If the latter phases vary from one near-field point to another, the character of constructive or destructive interference also varies in space and may lead to enhanced light intensity at one point and decreased intensity at another point within the extent of the near-field mode. The possibility for control now arises because the external phases $\varphi_{1}(\omega)$ and $\varphi_{2}(\omega)$ can be modulated at will, thereby choosing at which place constructive and at which place destructive interference will occur. With this explanation in mind, it is also clear why the optimization with phase-only shaped pulses of Figs. 9(c) and 9(d) was not

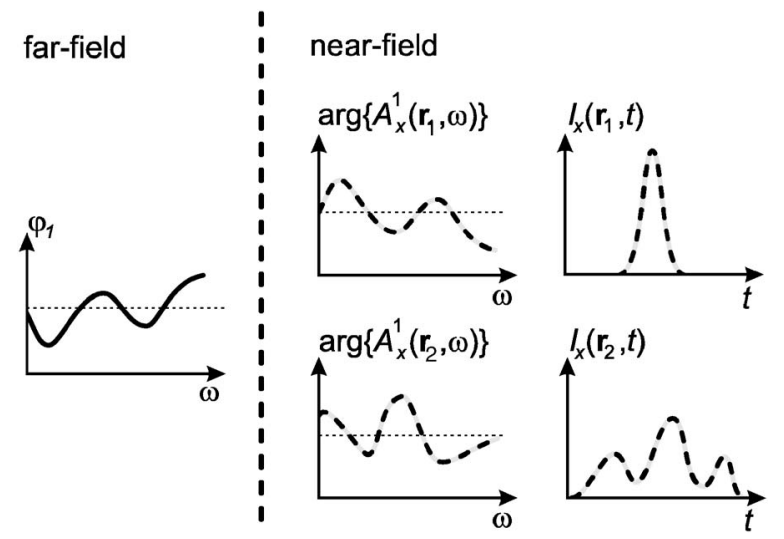

FIG. 11. Local pulse compression scheme. The local phase $\arg \left\{A_{x}^{1}(\mathbf{r}, \omega)\right\}$ in the optical near-field distribution depends on location $\mathbf{r}$. Thus, at some location bandwidth-limited pulses can be achieved by adjusting the external phase, whereas at another location the light pulse is in general stretched.

successful: Although the interference between the modes $\mathbf{E}_{\text {local }, 1}$ and $\mathbf{E}_{\text {local }, 2}$ occurs, it was not possible to influence at which point it will be constructive and at which point destructive because the phases $\varphi_{1}(\omega)$ and $\varphi_{2}(\omega)$ are coupled for linearly polarized light fields.

This mechanism of two-pathway interference is extended further by considering the broadband character of femtosecond light pulses. For each separately controllable wavelength component (as determined by the pixel number and resolution of the pulse-shaping device), interference can be adjusted individually. One of the advantages of using the boundary-element method for electric-field calculations is that the near-field response is directly obtained in the frequency domain. This has the consequence that the control mechanism discussed here is immediately visible, at least on a qualitative level. As mentioned above, having the near-field response available in the frequency domain is also important for quantitative optimization of specific properties. In this way, the time-consuming solution of Maxwell's equation has to be obtained only once, and the varying effects of different pulse shapes can be calculated via Eq. (3) by simple multiplication.

\section{B. Local pulse compression}

A second control mechanism is important for those observables in which the temporal properties of the local field are important (e.g., control of nonlinear flux or spatialtemporal distributions). For a schematic illustration, consider first only one external polarization component (say 1), but with broadband spectrum, and its effect on the near field (Fig. 11). Assume that the objective is to create a compressed pulse at position $\mathbf{r}_{1}$ in the near field (Fig. 11 upper right panel). For simplicity, also consider just the $x$ component. The near-field response then has a characteristic spectral phase structure $\arg \left\{A_{x}^{1}\left(\mathbf{r}_{1}, \omega\right)\right\}$ at position $\mathbf{r}_{1}$ (Fig. 11 upper middle panel). It is known from femtosecond pulse generation that the shortest possible pulses are obtained with flat spectral phase. Hence, in order to achieve the desired tem- 
poral peak, one has to choose a far-field phase $\varphi_{1}(\omega)$ [Fig. 11 left panel] that is exactly the negative of the near-field phase response $\arg \left\{A_{x}^{1}\left(\mathbf{r}_{1}, \omega\right)\right\}$, such that the sum delivers the required flat phase according to Eq. (3). This discussion is completely analogous to the dispersion compensation experiments that have been carried out with pulse shapers for many years. ${ }^{38,39}$

However, the difference to ordinary far-field compensation techniques emerges when considering the spatial variation of $\arg \left\{A_{x}^{1}\left(\mathbf{r}_{1}, \omega\right)\right\}$. At a different position $\mathbf{r}_{2}$, the phase response $\arg \left\{A_{x}^{1}\left(\mathbf{r}_{2}, \omega\right)\right\}$ may also be different (Fig. 11, lower middle panel). The sum of external and near-field phases is then not necessarily flat any more, and the resulting temporal intensity evolution is not a short pulse but can be spread out (lower right panel). Hence, while at location $\mathbf{r}_{1}$ a high intensity and correspondingly large values for nonlinear signals may be achieved, the time-integrated squared intensity is lower at $\mathbf{r}_{2}$. By varying the external phase, it is hence possible to localize nonlinear signals spatially. Of course if the external phase is chosen to be the inverse of the near-field phase at $\mathbf{r}_{2}$ instead of at $\mathbf{r}_{1}$, the nonlinear signal can be moved accordingly.

The possibilities for control increase further by considering that not only one external polarization component but both of them can be exploited as control parameters in this fashion. In addition, one has to take into account that the electric near field has three polarization components whose near-field phase responses $\arg \left\{A_{\alpha}^{1}(\mathbf{r}, \omega)\right\}, \alpha=x, y, z$, are in general not identical.

The general control scheme exploits both of the mechanisms, local mode interference (Sec. IV A) and local pulse compression (Sec. IV B). It is the detailed interplay between these two and their generalization to all frequencies and polarization directions that makes the very flexible control possible that was shown in previous sections. Because of the complex details of the spatially varying near-field response, a learning algorithm was used for automated optimization of desired control objectives. The underlying basic mechanisms, however, can be understood also in these simplified pictures.

\section{CONCLUSION AND OUTLOOK}

In this work we have introduced and analyzed theoretically a technique for ultrafast adaptive control of optical near fields. By combining polarization shaping of femtosecond light pulses with the near-field properties of nanostructures, many possibilities arise both in the areas of time-/spaceresolved spectroscopy as well as in quantum control.

As a basic calculation procedure for near-field responses we employ the boundary-element method that delivers a rigorous solution of Maxwell's equations in the frequency domain. The only assumption is that material behavior can be described by a frequency-dependent complex-valued dielectric function. Apart from this, the full vectorial and temporal character of the irradiating femtosecond light pulses is incorporated along with propagation/retardation effects. As a result, the three-dimensional temporal evolution of electromagnetic near fields can be calculated at any point in the vicinity of a nanostructure under irradiation with arbitrarily complex and polarization-shaped femtosecond laser pulses.

The resulting field is analyzed in terms of a number of different observables which in turn are used to define optimization goals. Employing a learning evolutionary algorithm, the external field properties are modified such that flexible control over the near field is achieved on a nanometer length scale. For example, we have demonstrated successfully the control over nanoscale spatial localization of linear and nonlinear flux, the generation of specific localized spectral shapes, and user-defined spatial-temporal intensity evolution.

Two dominant control mechanisms were identified. One important scheme is the interference of near-field modes that are excited by the two external polarization components. Hence it is essential to use polarization-modulated input pulses for full near-field control, whereas linearly polarized phase-shaped laser pulses cannot access this mechanism. Additionally, local pulse compression can be exploited such that the desired temporal structure is created at a specified nanoscale location.

Similar to SNOM our scheme relies on the local field enhancement and the spatially inhomogeneous field distribution near nanostructures. However, one of the difficulties in SNOM experiments arises from the fact that the "nanostructure" consisting of scanning tip and the actual nanostructure under investigation is continuously changing in the scanning operation. This may lead to topographic artifacts and optical near-field distributions that are not directly evident. In the schemes proposed in our paper, this is different: the nanostructure is static and any change in the response of the illuminated nanostructure is connected directly to the near field distribution.

This methodology of near-field shaping opens the route toward controlled nano-optics and spectroscopy and therefore to a wide range of applications. For example, near-field scanning techniques could benefit from the ability to tailor electric-field properties in the interaction region, optimizing contrast and nonlinear response. Spatial localization can furthermore be implemented as an ultrafast nanooptical switch, delivering tightly confined (in space and time) "packets" of electromagnetic energy at desired locations below the diffraction limit. Thus an optical multiplexer can be realized that does not require processing time. This might also have applications in quantum information processing because it would enable the addressing and manipulation of nanolocalized quantum registers (qubits). Another field of applications emerges in the area of plasmonic transport phenomena where near-field shaping may allow the control over signal propagation in confined geometries.

In the area of quantum control, it is possible to create light fields with complex time evolution along three polarization directions. Such light fields have potential applications in molecular coherent control ${ }^{42}$ and, in particular, chirality control. ${ }^{43-45}$ More generally speaking, three-dimensionally shaped light fields offer the possibility for controlling vectorial properties of light-matter interaction on a fundamental level. Furthermore, the field properties can be changed on length scales smaller than extended quantum wave functions. This should cause fascinating effects as the conventionally 
assumed dipole approximation breaks down, i.e., the electric field is not constant any more throughout the size of the investigated system.

Finally, nanoscopic ultrafast space-time-resolved spectroscopy should be feasible where pump and probe interactions do not only occur at different times but also at different positions. This would enable the direct spatial probing of nanoscale energy transfer or charge transfer processes and give us an immediate view into coupling mechanisms of complex quantum systems such as macromolecules or quantum-dot assemblies.

Optical near-field control is a research direction that is just emerging, with a lot of potential in fundamental and applied sciences. Just as adaptive pulse shaping with learning algorithms and experimental feedback has brought a breakthrough to the area of chemical reaction control, we believe that adaptive shaping of electromagnetic near fields can be of similar value in nanophotonics.

\section{ACKNOWLEDGMENS}

T.B. thanks the German Science Foundation for their support. F.J.G.A. acknowledges help and support from the Spanish MEC (FIS2004-06490-C03-02).
${ }^{1}$ D. W. Pohl, W. Denk, and M. Lanz, Appl. Phys. Lett. 44, 651 (1984).

${ }^{2}$ K. Kneipp, Y. Wang, H. Kneipp, L. T. Perelman, I. Itzkan, R. R. Dasari, and M. S. Feld, Phys. Rev. Lett. 78, 1667 (1997).

${ }^{3}$ W. L. Barnes, A. Dereux, and T. W. Ebbesen, Nature (London) 424, 824 (2003).

${ }^{4}$ T. W. Ebbesen, H. J. Lezec, H. F. Ghaemi, T. Thio, and P. A. Wolff, Nature (London) 391, 667 (1998).

${ }^{5}$ M. Quinten, A. Leitner, J. R. Krenn, and F. R. Aussenegg, Opt. Lett. 23, 1331 (1998).

${ }^{6}$ S. A. Maier, P. G. Kik, H. A. Atwater, S. Meltzer, E. Harel, B. E. Koel, and A. A. G. Requicha, Nat. Mater. 2, 229 (2003).

${ }^{7}$ E. J. Sanchez, L. Novotny, and X. S. Xie, Phys. Rev. Lett. 82, 4014 (1999).

${ }^{8}$ S. I. Bozhevolnyi, J. Beermann, and V. Coello, Phys. Rev. Lett. 90, 197403 (2003).

${ }^{9}$ D. S. Kim, S. C. Hohng, V. Malyarchuk, Y. C. Yoon, Y. H. Ahn, K. J. Yee, J. W. Park, J. Kim, Q. H. Park, and C. Lienau, Phys. Rev. Lett. 91, 143901 (2003).

${ }^{10}$ M. Merschdorf, C. Kennerknecht, and W. Pfeiffer, Phys. Rev. B 70, 193401 (2004).

${ }^{11}$ M. Munzinger, C. Wiemann, M. Rohmer, L. Guo, M. Aeschlimann, and M. Bauer, New J. Phys. 7, 68 (2005).

${ }^{12}$ K. Atsushi, K. Onda, H. Petek, Z. Sun, Y. S. Jung, and H. K. Kim, Nano Lett. 5, 1123 (2005).

${ }^{13}$ M. I. Stockman, S. V. Faleev, and D. J. Bergman, Phys. Rev. Lett. 88, 067402 (2002).

${ }^{14}$ D. J. Tannor and S. A. Rice, J. Chem. Phys. 83, 5013 (1985).

${ }^{15}$ P. Brumer and M. Shapiro, Chem. Phys. Lett. 126, 541 (1986).

${ }^{16}$ D. J. Tannor, R. Kosloff, and S. A. Rice, J. Chem. Phys. 85, 5805 (1986).

${ }^{17}$ A. P. Peirce, M. A. Dahleh, and H. Rabitz, Phys. Rev. A 37, 4950 (1988)

${ }^{18}$ U. Gaubatz, P. Rudecki, M. Becker, S. Schiemann, M. Külz, and K. Bergmann, Chem. Phys. Lett. 149, 463 (1988).

${ }^{19}$ R. Kosloff, S. A. Rice, P. Gaspard, S. Tersigni, and D. J. Tannor, Chem. Phys. 139, 201 (1989).

${ }^{20}$ T. Brixner and G. Gerber, ChemPhysChem 4, 418 (2003).

${ }^{21}$ R. S. Judson and H. Rabitz, Phys. Rev. Lett. 68, 1500 (1992).

${ }^{22}$ T. Brixner and G. Gerber, Opt. Lett. 26, 557 (2001).

${ }^{23}$ T. Brixner, G. Krampert, P. Niklaus, and G. Gerber, Appl. Phys. B: Lasers Opt. 74, S133 (2002).

${ }^{24}$ T. Brixner, Appl. Phys. B 76, 531 (2003).
${ }^{25}$ T. Brixner, N. H. Damrauer, G. Krampert, P. Niklaus, and G. Gerber, J. Opt. Soc. Am. B 20, 878 (2003).

${ }^{26}$ D. Oron, N. Dudovich, and Y. Silberberg, Phys. Rev. Lett. 90, 213902 (2003).

${ }^{27}$ N. Dudovich, D. Oron, and Y. Silberberg, Phys. Rev. Lett. 92, 103003 (2004).

${ }^{28}$ T. Suzuki, S. Minemoto, T. Kanai, and H. Sakai, Phys. Rev. Lett. 92, 133005 (2004).

${ }^{29}$ T. Brixner, G. Krampert, T. Pfeifer, R. Selle, G. Gerber, M. Wollenhaupt, O. Graefe, C. Horn, D. Liese, and T. Baumert, Phys. Rev. Lett. 92, 208301 (2004).

${ }^{30}$ Y. Silberberg, Nature (London) 430, 624 (2004).

${ }^{31}$ T. Brixner, W. Pfeiffer, and F. J. García de Abajo, Opt. Lett. 29, 2187 (2004).

${ }^{32}$ T. Brixner, F. J. García de Abajo, J. Schneider, and W. Pfeiffer, Phys. Rev. Lett. 95, 093901 (2005).

${ }^{33}$ F. J. García de Abajo and A. Howie, Phys. Rev. Lett. 80, 5180 (1998).

${ }^{34}$ F. J. García de Abajo and A. Howie, Phys. Rev. B 65, 115418 (2002).

${ }^{35}$ E. D. Palik, Handbook of Optical Constants of Solids (Academic, New York, 1997).

${ }^{36} \mathrm{U}$. Kreibig and M. Vollmer, Optical properties of metal clusters (Springer, Berlin, 1995).

${ }^{37}$ A. Hohenau, J. R. Krenn, G. Schider, H. Ditlbacher, A. Leitner, F. R. Aussenegg, and W. L. Schaich, Europhys. Lett. 69, 543 (2005).

${ }^{38}$ A. M. Weiner, Rev. Sci. Instrum. 71, 1929 (2000).

${ }^{39}$ T. Baumert, T. Brixner, V. Seyfried, M. Strehle, and G. Gerber, Appl. Phys. B 65, 779 (1997).

${ }^{40}$ T. Brixner, F. J. García de Abajo, C. Spindler, and W. Pfeiffer Appl. Phys. B (to be published).

${ }^{41}$ T. Brixner, J. Schneider, W. Pfeiffer, and F. J. García de Abajo, in Ultrafast Phenomena XIV, edited by T. Kobayashi, T. Okada, T. Kobayashi, and S. De Silvestri (Springer, Berlin, 2005) p. 670.

${ }^{42}$ R. Wu, I. R. Sola, and H. Rabitz, Chem. Phys. Lett. 400, 469 (2004).

${ }^{43}$ E. Frishman, M. Shapiro, D. Gerbasi, and P. Brumer, J. Chem. Phys. 119, 7237 (2003).

${ }^{44}$ K. Hoki, L. Gonzáles, and Y. Fujimura, J. Chem. Phys. 116, 8799 (2002).

${ }^{45}$ S. S. Bychkov, B. A. Grishanin, and V. N. Zadkov, J. Exp. Theor. Phys. 93, 24 (2001). 